Z Rheumatol $2016 \cdot 75: 54-62$

DOI 10.1007/s00393-015-0031-9

Online publiziert: 19. Januar 2016

○) Springer-Verlag Berlin Heidelberg 2015

Redaktion

H.-P. Tony, Würzburg

CrossMark

\author{
M. Boegemann ${ }^{1} \cdot$ R. Alten ${ }^{2} \cdot$ M. Aringer ${ }^{3} \cdot$ S. Zastrow ${ }^{4}$ \\ 'Klinik für Urologie und Kinderurologie, Universitätsklinikum Münster, Münster, Deutschland \\ ${ }^{2}$ Innere Medizin II - Rheumatologie, Schlossparkklinik Universitätsmedizin Berlin, Berlin, Deutschland \\ ${ }^{3}$ Medizinische Klinik III, Bereich Rheumatologie, Universitätsklinikum Carl Gustav Carus Dresden, Dresden, \\ Deutschland \\ ${ }^{4}$ Klinik und Poliklinik für Urologie, Universitätsklinikum Carl Gustav Carus Dresden, Dresden, Deutschland
}

\title{
Urologische Malignome und rheumatoide Arthritis
}

\section{Epidemiologie und Standard- therapie urologischer Tumoren}

\section{Prostatakarzinom}

Das PCa ist eines der weltweit häufigsten Malignome, besonders in alternden Populationen. Im Jahr 2010 erkrankten in Deutschland geschätzt 164,2/100.000 Männer; 31,6/100.000 Männer verstarben am PCa [2]. Demografische Daten $\mathrm{zu}$ allen beschriebenen urologischen $\mathrm{Tu}$ morentitäten sind in $\bullet$ Tab. 1 zusammengefasst.

Bei Erstdiagnose eines PCa steht in Abhängigkeit vom Alter die Ausbreitungsdiagnostik im Vordergrund. Angelehnt an die S3-Leitlinie zum PCa [1], ergeben sich folgende Behandlungsoptionen: Bei lokal begrenzten PCa kann eine kurative Therapie per radikaler Prostatektomie oder definitiver Radiatio erfolgen. $\mathrm{Ab}$ einer zu erwartenden verbleibenden Lebenserwartung von unter 10 Jahren kann in Absprache mit dem Patienten statt einer invasiven Therapie auch „watchful waiting“ betrieben werden. Bei sog. Low-risk-PCa (PSA $<10 \mathrm{ng} / \mathrm{ml}, \leq 2$ positive Stanzzylinder, Gleason-Score $\leq 6$ und PCa nicht tast- bar), die bei nur ca. $5 \%$ der betroffenen Männer zum Tod durch das PCa führen [25], stellt die aktive Überwachung eine Alternative dar. Dabei wird regelmäßig durch Tastuntersuchung, Überprüfen des PSA-Verlaufs und Prostatabiopsie kontrolliert, ob sich die Charakteristika des PCa verändern.

Hormonentzug (Übersicht der bei urologischen Malignomen eingesetzten Substanzen in - Tab. 2) kann den Verlauf nur hinauszögern. Praktisch alle Männer, die an einem PCa versterben, versterben im Stadium des kastrationsresistenten Prostatakarzinoms (CRPC) mit metastasierter Erkrankung. Das CRPC-Stadium wird unter Hormonentzug früher oder später immer erreicht. Beim CRPC wird palliativ, aber teils mit der Möglichkeit zur Überlebenszeitverlängerung mittels Next-generation-Hormonentzugstherapie (Abirateronacetat, Enzalutamid), Chemotherapie (Docetaxel, Cabazitaxel) oder Radionukliden (z. B. Radium223) therapiert. Soweit aktuell absehbar, wird eine immunmodulierende Therapie beim PCa keine nennenswerte Rolle spielen. Hingegen wird parallel zur Therapie mit Abirateronacetat und taxanhaltiger Chemotherapie regelhaft eine zellkarzinomen (RCC) [23], Urothelkarzinomen (TCC) [22] und Prostatakarzinomen (PCa) [14] sowie auch einer seropositiven RA [7]. Dieser Umstand und der demografische Wandel erhöhen die Wahrscheinlichkeit, dass Rheumatologen Patienten mit zeitgleich bestehenden urologischen Tumorerkrankungen behandeln müssen.

\begin{tabular}{|lllll|}
\hline Tab. 1 Daten RKI (Robert Koch-Institut) Krebs in Deutschland 2010 [2] \\
\hline Malignom & \multicolumn{2}{l}{ Inzidenz/100.000 } & $\begin{array}{l}\text { Mittleres } \\
\text { Erkrankungsalter }\end{array}$ & $\begin{array}{l}\text { 5-Jahres-Überleben } \\
\text { (\%) }\end{array}$ \\
\cline { 2 - 5 } & Männer & Frauen & & 78 \\
\hline Prostatakarzinom & 164,2 & - & 70 & 67 \\
\hline Nierenzellkarzinom & 22,3 & 13,4 & 70 & 44 \\
\hline Urothelkarzinom & 28,3 & 10,0 & 75 & 95 \\
\hline $\begin{array}{l}\text { Keimzelltumoren } \\
\text { Hoden }\end{array}$ & 9,7 & - & 38 & \\
\hline
\end{tabular}


Tab. 2 Systemische Therapien urologischer Malignome

\begin{tabular}{|c|c|c|c|c|}
\hline Tumorentität & Wirkstoff & Wirkmechanismus & Applikation/Frequenz & $\begin{array}{l}\text { Wichtig für Rheumato- } \\
\text { logen }\end{array}$ \\
\hline \multirow[t]{7}{*}{ Prostatakarzinom } & $\begin{array}{l}\text { LHRH-Analoga } \\
\text { GnRH-Antagonisten }\end{array}$ & $\begin{array}{l}\text { Testikuläre Hormonentzugs- } \\
\text { therapie }\end{array}$ & s. c. 1-bis 6-monatlich & \\
\hline & $\begin{array}{l}\text { Bicalutamid } \\
\text { Flutamid } \\
\text { Cyproteronacetat }\end{array}$ & 1.-Generation-Antiandrogene & p. o. täglich & \\
\hline & $\begin{array}{l}\text { Abirateronacetat + Pred- } \\
\text { nison }\end{array}$ & Androgensyntheseinhibitor & p.o. täglich & Prednison $5 \mathrm{mg} 1-0-1$ \\
\hline & Enzalutamid & 2.-Generation-Antiandrogen & p.o. täglich & \\
\hline & $\begin{array}{l}\text { Docetaxel } \\
\text { Cabazitaxel }\end{array}$ & Klassische Zytostatika & i. v. verschiedene Regime & $\begin{array}{l}\text { Prednison } 5 \text { mg 1-0-1, } \\
\text { kurzfristig Dexametha- } \\
\text { son }\end{array}$ \\
\hline & Radium-223 & a-Emitter & i. v. 6 Zyklen à 4 Wochen & \\
\hline & PSMA-Liganden & $a-/ \beta$-Emitter & i.v. ca. 6- bis 8-wöchentlich & \\
\hline \multirow[t]{4}{*}{ Urothelkarzinom } & Cisplatin + Gemcitabine & Klassische Zytostatika & $\begin{array}{l}\text { i. v. } 2 \text { bis } 6 \text { Zyklen à } 3 \text { bis } 4 \text { Wo- } \\
\text { chen }\end{array}$ & $\begin{array}{l}\text { Kurzfristig Dexametha- } \\
\text { son }\end{array}$ \\
\hline & Vinflunine & Klassisches Zytostatikum & i. v. 3 bis 6 Zyklen à 3 Wochen & $\begin{array}{l}\text { Kurzfristig Dexametha- } \\
\text { son }\end{array}$ \\
\hline & Pembrolizumab ${ }^{a}$ & PD-1-Antikörper & i.v. 3-wöchentlich & Immunmodulation \\
\hline & Bacillus Calmette-Guérin & Immunmodulation & Intravesikal & Immunmodulation \\
\hline \multirow[t]{4}{*}{ Nierenzellkarzinom } & $\begin{array}{l}\text { Sorafenib } \\
\text { Sunitinib } \\
\text { Pazopanib } \\
\text { Axitinib } \\
\text { Cabozantinib }^{\text {a }}\end{array}$ & Tyrosinkinaseinhibitoren & p. o. verschieden & \\
\hline & $\begin{array}{l}\text { Everolimus } \\
\text { Temsirolimus }\end{array}$ & mTOR-Inhibitoren & $\begin{array}{l}\text { p. o. täglich } \\
\text { i.v. wöchentlich }\end{array}$ & Immunmodulation \\
\hline & $\begin{array}{l}\text { Bevacizumab + a-Interfe- } \\
\text { ron }\end{array}$ & VEGF-Antikörper & i. v./s.c. & Immunmodulation \\
\hline & $\begin{array}{l}\text { Nivolumab } \\
\text { Ipilimumab }^{\mathrm{a}}\end{array}$ & $\begin{array}{l}\text { PD-1-Antikörper } \\
\text { CTLA-4-Antikörper }\end{array}$ & $\begin{array}{l}\text { i.v. Nivolumab 2-wöchentlich, } \\
\text { i.v. Ipilimumab } 12 \text { Wochen }\end{array}$ & Immunmodulation \\
\hline \multirow[t]{3}{*}{ Keimzelltumor } & $\begin{array}{l}\text { Cisplatin + Etoposid + } \\
\text { Bleomycin } \\
\text { Cisplatin + Etoposid + } \\
\text { Ifosfamid (Mesna) }\end{array}$ & Klassische Zytostatika & i. v./1 bis 4 Zyklen à 3 Wochen & $\begin{array}{l}\text { Kurzfristig Dexametha- } \\
\text { son } \\
\text { Cave: Urothelkarzinome }\end{array}$ \\
\hline & Carboplatin & Klassisches Zytostatikum & i.v./Einmalgabe & \\
\hline & $\begin{array}{l}\text { Hochdosisprotokolle (sehr } \\
\text { selten) }\end{array}$ & Klassische Zytostatika & i. v./sehr verschieden & $\begin{array}{l}\text { Extreme Aplasie/ } \\
\text { Stammzellreplantation }\end{array}$ \\
\hline
\end{tabular}

LHRH luteinisierendes Hormon Releasing-Hormon, PSMA prostataspezifisches Membranantigen, GnRH Gonadotropin-Releasing-Hormon

${ }^{a}$ Noch nicht zugelassen, aber Zulassung bevorstehend oder sehr wahrscheinlich.

entzündungshemmende Begleittherapie mit niedrig dosierten Glukokortikoiden durchgeführt.

\section{Urothelkarzinom}

In Deutschland kam es im Jahr 2010 zu 28,3 Neuerkrankungen eines TCC pro 100.000 Männern und 10,0/100.000 Frauen; 9,1/100.000 Männer und 4,5/ 100.000 Frauen mit einem TCC verstarben daran [2]. Bei organbegrenzten TCC (70\%) ohne Muskelinfiltration (< pT2) kann bei $90 \%$ der Patienten mittels transurethraler Harnblasentumorresektion eine Erkrankungskontrolle erreicht werden, teilweise mithilfe von intravesikalen Chemo- oder Immuntherapeutika. Bei den $10 \%$ der organbegrenzten Karzinome, bei denen dies nicht gelingt, und bei $30 \%$ der erstdiagnostizierten TCC mit Muskelinvasion kann nur zu $50 \%$ mittels radikaler Zystektomie und Harnableitung eine Heilung erreicht werden. Die deutliche Mehrzahl der Patienten, die an einem TCC versterben, verstirbt im metastasierten Erkrankungsstadium. In diesem Stadium kann eine palliative Chemotherapie (Cisplatin/Gemcitabine, Vinflunine), perspektivisch aber auch eine Immuntherapie mit PD-1-, PD-L1oder CTLA-4-Antagonisten durchgeführt werden.

\section{Nierenzellkarzinom}

Es gibt drei 3 Haupttypen des RCC mit spezifischen genetischen und prognostischen Charakteristika. Am häufigsten ist 
das klarzellige RCC, seltener sind die papillären und chromophoben Subtypen.

Im Jahr 2010 wurde in Deutschland die Inzidenz des RCC auf 22,3/100.000 Männer und 13,4/100.000 Frauen geschätzt [2]. Demgegenüber standen 7,7/100.000 Todesfälle bei Männern und 5,2/100.000 bei Frauen.

Die primäre Therapie des lokalisierten organbegrenzten RCC besteht in einer nierenerhaltenden Tumorresektion oder, wenn nicht möglich, in der Tumornephrektomie.

Die Prognose nach operativer Therapie hängt von den pathohistologischen Charakteristika und bestimmten klinischen Faktoren, wie z. B. dem ECOGPerformance-Status, ab. Für die Integration dieser Parameter wurden Vorhersagemodelle entwickelt. Exemplarisch kann das tumorspezifische Überleben an einem sehr gut validierten Nomogramm bestimmt werden [16]. Für einen kleinen $(2 \mathrm{~cm})$, inzidentell entdeckten lokalisierten G2-Tumor ohne Symptomatik ist die tumorspezifische Überlebenswahrscheinlichkeit nach 5 Jahren mit $98 \%$ hoch. Für einen symptomatischen (Makrohämaturie) G3-Tumor von $6 \mathrm{~cm}$ Durchmesser mit Thrombosierung in der V. cava inferior, jedoch ohne lymphogene oder Fernmetastasen läge die tumorspezifische 5-Jahres-Überlebenswahrscheinlichkeit nach Operation bei noch immer etwa $60 \%$.

Im metastasierten Stadium können durch den sequenziellen Einsatz moderner Therapeutika mediane Gesamtüberlebenszeiten von 30 Monaten erreicht werden $[11,20]$.

\section{Keimzelltumoren}

Man unterscheidet Seminome (SKZT) und Nichtseminome (NSKZT) bzw. Mischtumoren mit Anteilen beider Histologien. Maligne Keimzelltumoren (KZT) machen 0,7\% aller malignen Neubildungen beim Mann aus [12]. Die Neuerkrankungsrate lag 2010 in Deutschland bei 9,7/100.000 Männer, die Mortalitätsrate bei 0,4/100.000 Männern [2]. Auf 10.000 geschätzte Todesfälle werden 2012 lediglich 2200 Fälle den mehr entwickelten Weltregionen zugeschrieben [12].

Z Rheumatol 2016 · 75:54-62 DOI 10.1007/s00393-015-0031-9

(c) Springer-Verlag Berlin Heidelberg 2015

\section{Boegemann $\cdot$ R. Alten $\cdot$ M. Aringer $\cdot$ S. Zastrow}

\section{Urologische Malignome und rheumatoide Arthritis}

\section{Zusammenfassung}

Urologische Malignome sind relativ häufig und nehmen mit zunehmendem Lebensalter zu. Der demografische Wandel führt dazu, dass bei immer mehr Patienten mit entzündlich-rheumatischen Erkrankungen urogenitale Tumoren koinzident sind oder unter antirheumatischer Therapie auftreten. Die Basistherapie rheumatischer Erkrankungen muss in dieses Fällen individuell sorgfältig abgewogen werden, obwohl die begrenzte Evidenz für die meisten Situationen gegen eine massive Risikoerhöhung durch konventionelle und/oder biologische Basistherapeutika spricht. Paraneoplastische Phänomene auch im Bewegungsapparat können durch urogenitale Malignome ausgelöst werden. Mit den sich abzeichnenden Umwälzungen der medikamentösen Tumortherapie durch die Immunonkologie ergeben sich darüber hinaus neue Herausforderungen für die interdisziplinäre Kooperation von Uroonkologen und Rheumatologen. In dieser Übersichtsarbeit werden unter Berücksichtigung der aktuellen deutschen und europäischen Leitlinien die Diagnostik, Standardbehandlung und Nachsorge urologischer Malignome skizziert. Wir beschreiben Kernaspekte, die von Urologen und Rheumatologen beachtet werden sollten. Durch persönliche Absprache, regelmäßigen Erfahrungsaustausch und Einbeziehung von Rheumatologen in interdisziplinäre Tumorboards sollte zukünftig eine bessere Behandlung unserer gemeinsamen Patienten möglich werden.

\section{Schlüsselwörter}

Paraneoplasie - Rheumatische Erkrankungen . Disease-modifying anti-rheumatic drugs . Immunsuppression - Tumorboard

\section{Genitourinary malignancies and rheumatoid arthritis}

\section{Abstract}

Genitourinary neoplasms are relatively common and the frequency increases with age. Due to demographic changes more patients with inflammatory rheumatic diseases will have concomitant genitourinary tumors or they will develop them under antirheumatic therapy. In such cases, diseasemodifying antirheumatic drugs (DMARD) and immunosuppressive therapy have to be carefully balanced on an individual basis. Based on the limited evidence available large increases in the risks from conventional and/or biological DMARDs for patients with genitourinary malignancies appear to be unlikely for most situations. In addition to these more common situations paraneoplastic symptoms in the musculoskeletal system can occur due to genitourinary malignancies. Moreover, novel drugs with immunostimulating activity for some geni- tourinary tumors may provoke autoimmune symptoms and thus present new challenges for interdisciplinary cooperation between rheumatologists and uro-oncologists. In this review, the diagnostic procedures, therapies and follow-up of cancers in the field of urology are delineated according to the current German and European guidelines. We describe the core issues that both urologists and rheumatologists should bear in mind. Direct communication, routine exchange and involvement of rheumatologists in interdisciplinary tumor boards should improve future treatment quality of our joint patients.

\section{Keywords}

Paraneoplastic syndromes · Rheumatic diseases - Disease-modifying antirheumatic drugs · Immunosuppression · Tumor board
Die Primärtherapie besteht in der Entfernung des befallenen Hodens. Anschließend wird bei nicht metastasierten Tumoren in Abhängigkeit von Histologie und Risikostratifizierung eine adjuvante Chemotherapie oder eine aktive Überwachung angeboten. In Einzelfällen kann beim NSKZT auch eine retroperitoneale Lymphknotenentfernung angeboten werden. In metastasierten Stadien wird eine primäre cisplatinhaltige Chemotherapie durchgeführt. Metastasenresiduen nach Chemotherapie werden beim 
Tab. 3 Wichtige Paraneoplasien bei urologischen Malignomen

Polymyalgia rheumatica und/oder Arteriitis temporalis

(Seronegative) rheumatoide Arthritis

„Relapsing seronegative symmetrical synovitis with pitting edema" (RS3PE)

Dermatomyositis und Polymyositis

„Scleroderma-like syndrome“

NSKZT operativ entfernt, beim SKZT lediglich im Verlauf kontrolliert [3].

Über alle Stadien gesehen, werden 5-Jahres-Überlebensraten von $97 \%$ erreicht. In metastasierten Stadien liegt diese immerhin noch zwischen 48 und $92 \%$ [3].

\section{Screening auf De-novo- Tumoren in der Urologie}

\section{Prostatakarzinom}

Ein bevölkerungsbasiertes Screening auf ein PCa bleibt auch heute, 25 Jahre nach Einführung des PSA-Tests, umstritten $[5,27]$. Einer vergleichsweise geringen Reduktion des PCa-spezifischen Überlebens steht einer eher hohen Rate an Überdiagnose und Übertherapie gegenüber.

Die S3-Leitlinie empfiehlt, dass mindestens 45 Jahre alte Männer, die eine mutmaßliche Lebenserwartung von mehr als 10 Jahren haben, über die Möglichkeit einer Früherkennung informiert werden sollen [1]. Die Männer sollen über Vor- und Nachteile der Früherkennung aufgeklärt werden, insbesondere über die Aussagekraft von positiven und negativen PSA-Testergebnissen und daraus abzuleitenden weiteren Maßnahmen. Diese Beratung sollte durch den Urologen erfolgen. In diesem Zusammenhang ist auf das mögliche Auftreten von paraneoplastischen rheumatischen Symptomen in Zusammenhang mit PCa hinzuweisen (•Tab. 3; [15, 21]).

\section{Urothelkarzinom}

Im Gegensatz zum PCa existiert für das TCC kein etablierter Screeningtest. Zur Diagnose eines TCC stehen auch heute die Klinik und Anamnese im Vorder- grund. Das Kardinalsymptom ist weiter die schmerzlose Makrohämaturie, die immer einen fachurologischen Tumorausschluss der ableitenden Harnwege nach sich ziehen sollte. Auch ein irritativer Harndrang kann ein Symptom eines TCC sein. Eine oft asymptomatische Hydronephrose kann ein Spätsymptom darstellen.

\section{》) Kardinalsymptom ist die schmerzlose Makrohämaturie}

Bei rheumatologischer Therapie mit TCC-fördernder Wirkung (z. B. Cyclophosphamid) sollte z. B. 3-monatlich ein Urinstatus erhoben werden. Bei erstmaligem Nachweis einer Hämaturie sollte bei Ausschluss einer Infektion und bei kurzfristiger Bestätigung des Befundes eine urologische Vorstellung erfolgen. Hiermit besteht eine Chance zur Früherkennung eines TCC. Insbesondere ehemaliger und aktiver Nikotinabusus geht mit einem TCC einher. Paraneoplasien sind beim TCC selten [26].

\section{Nierenzellkarzinom}

Ein einfach einzusetzender Screeningtest für RCC existiert nicht. Im Rahmen von nicht durch die gesetzliche Krankenversicherung (GKV) getragenen Vorsorgeuntersuchungen kann die Ultraschalluntersuchung des Abdomens Hinweise auf RCC ergeben. Am häufigsten werden RCC aber mittlerweile inzidentell im Rahmen bildgebender Untersuchungen aufgrund anderer Indikationen entdeckt. In einer aktuellen Serie beläuft sich der Anteil inzidentell entdeckter RCC auf $67 \%$ [18]. Die klassische Symptomtrias aus Flankenschmerz, Makrohämaturie und einem abdominellen Tumor findet sich lediglich in fortgeschrittenen Stadien. Paraneoplastische Syndrome sind beim RCC häufig [17]. Eine signifikante Assoziation mit Erkrankungen des rheumatischen Formenkreises ist jedoch für RCC nicht beschrieben.

\section{Keimzelltumoren}

KZT werden durch einen palpablen Tumor symptomatisch. Regelmäßige Selbst- untersuchungen junger Männer sind daher wünschenswert. Jede Hodenschwellung sollte umgehend urologisch abgeklärt werden. Systematische Screeningtests existieren nicht. Als Risikofaktoren für die Entstehung eines malignen KZT gelten ein Hodenhochstand im Kindesalter und eine positive Familienanamnese. Ist ein Patient bereits an einem Hoden wegen eines KZT behandelt worden, hat er ein deutlich erhöhtes Risiko für die Entwicklung eines Tumors auf der Gegenseite [3].

\section{Nachsorge urologischer Tumoren}

\section{Prostatakarzinom}

Laut S3-Leitlinie wird nach kurativer Therapie eine PSA-gestützte Nachsorge empfohlen [1]. Detaillierte Nachsorgeschemata zu den urologischen Malignomen sind in - Tab. 4 dargestellt.

Nach radikaler Prostatektomie kennzeichnet ein in mindestens 2 Messungen bestätigter PSA-Wert von $>0,2 \mathrm{ng} / \mathrm{ml}$ ein Lokalrezidiv. Nach definitiver Radiatio liegt ein Rezidiv vor, wenn der PSA-Wert mehr als 2 ng/ml über den Nadir nach Radiatio wieder ansteigt. Bei einer PSAVerdopplungszeit $<10$ Monate ist eine zwischenzeitlich entstandene Fernmetastasierung wahrscheinlich [24]. Wenn ein Lokalrezidiv wahrscheinlich ist, kann je nach vorheriger Therapie eine SalvageRadiatio oder eine radikale Prostatektomie vorgenommen werden, um jeweils eine Kuration zu erreichen. Eine routinemäßige bildgebende Nachsorge erfolgt nicht, sondern nur bei klinischem Verdacht auf eine Metastasierung oder wenn aus der Bildgebung eine Konsequenz erwachsen würde.

\section{Urothelkarzinom}

Die europäische Leitlinie zum nicht muskelinvasiven TCC der Harnblase sieht zur Nachsorge von Low-risk-TCC nach makroskopisch vollständiger Resektion die regelmäßige Zystoskopie vor [6]. Die Nachsorge von High-risk-TCC erfolgt engmaschiger. Zusätzlich zum Ausschluss von Malignomen im Ureter oder Nierenbecken sollte bei High-risk- 
Hier steht eine Anzeige.

算 Springer 
Tab. 4 Nachsorgeschemata urologischer Malignome nach kurativer Therapie

\begin{tabular}{|c|c|c|c|}
\hline Tumorentität & Jahr 1-2 & Jahr 3-4 & Ab Jahr 5 \\
\hline Prostatakarzinom [1] & PSA 3-monatlich & PSA halbjährlich & PSA jährlich \\
\hline Urothelkarzinom & Jahr 1 & Ab Jahr 2 & \\
\hline \multirow[t]{5}{*}{ Nicht muskelinvasiv [6] } & "Low risk" & & \\
\hline & Zystoskopie nach 3 und 9 Monaten & Zystoskopie jährlich & \\
\hline & Jahr 1-2 & Jahr 3-5 & Ab Jahr 6 \\
\hline & "High risk" & & \\
\hline & $\begin{array}{l}\text { Zystoskopie 3-monatlich } \\
\text { AUG oder Uro-MRT jährlich }\end{array}$ & $\begin{array}{l}\text { Zystoskopie halbjährlich } \\
\text { AUG oder Uro-MRT jährlich }\end{array}$ & $\begin{array}{l}\text { Zystoskopie jährlich } \\
\text { AUG oder Uro-MRT jährlich }\end{array}$ \\
\hline \multirow[t]{2}{*}{ Muskelinvasiv [29] } & Jahr 1-3 & Jahr 4-5 & \\
\hline & $\begin{array}{l}\text { Schnittbildgebung halbjährlich oder } \\
\text { individuell }\end{array}$ & $\begin{array}{l}\text { Schnittbildgebung jährlich oder } \\
\text { individuell }\end{array}$ & \\
\hline \multirow[t]{6}{*}{ Nierenzellkarzinom [19] } & Jahr 1 & Nach Jahr 3 & Nach Jahr 5 \\
\hline & "Low risk" & & \\
\hline & $\begin{array}{l}\text { Schnittbildgebung nach } 6 \text { Monaten } \\
\text { Sonographie }\end{array}$ & $\begin{array}{l}\text { Schnittbildgebung nach } 2 \text { Jahren } \\
\text { Sonographie }\end{array}$ & $\begin{array}{l}\text { Schnittbildgebung nach } 4 \text { Jahren } \\
\text { Sonographie }\end{array}$ \\
\hline & Jahr 1 & Jahr 1-5 & Ab Jahr 6 \\
\hline & "Intermediate" und "high risk" & & \\
\hline & Schnittbildgebung nach 6 Monaten & Schnittbildgebung jährlich & Schnittbildgebung 2-jährlich \\
\hline Keimzelltumor [3] & Jahr 1-2 & Jahr 3 & Jahr 4-5 \\
\hline \multirow[t]{2}{*}{ NSKZT } & Keine Metastasen & & \\
\hline & $\begin{array}{l}\text { Körperliche Untersuchung } \\
\text { Tumormarker } \\
\text { Schnittbildgebung nach 3, } 12 \text { und } \\
24 \text { Monaten } \\
\text { Thoraxröntgenaufnahme jährlich }\end{array}$ & $\begin{array}{l}\text { Körperliche Untersuchung } \\
\text { Tumormarker 3-monatlich } \\
\text { Schnittbildgebung nach } 36 \text { Monaten }\end{array}$ & $\begin{array}{l}\text { Körperliche Untersuchung } \\
\text { Tumormarker } \\
\text { Schnittbildgebung } \\
\text { Alles jährlich }\end{array}$ \\
\hline \multirow[t]{3}{*}{ SKZT } & Jahr 1-2 & Jahr 3-5 & \\
\hline & Keine Metastasen & & \\
\hline & $\begin{array}{l}\text { Körperliche Untersuchung } \\
\text { Tumormarker 4-monatlich } \\
\text { Schnittbildgebung, Thoraxröntgen- } \\
\text { aufnahme abwechselnd halbjährlich }\end{array}$ & $\begin{array}{l}\text { Körperliche Untersuchung } \\
\text { Tumormarker jährlich } \\
\text { Schnittbildgebung nach } 36 \text { und } \\
60 \text { Monaten }\end{array}$ & \\
\hline \multirow[t]{2}{*}{ Metastasierte KZT } & Jahr 1-2 & Jahr 3-5 & Ab Jahr 6 \\
\hline & $\begin{array}{l}\text { Körperliche Untersuchung } \\
\text { Tumormarker 3-monatlich } \\
\text { Schnittbildgebung, Thoraxröntgen- } \\
\text { aufnahme abwechselnd halbjährlich }\end{array}$ & $\begin{array}{l}\text { Körperliche Untersuchung } \\
\text { Tumormarker 6-monatlich } \\
\text { Schnittbildgebung, Thoraxröntgen- } \\
\text { aufnahme abwechselnd jährlich }\end{array}$ & Individuell \\
\hline
\end{tabular}

TCC jährlich eine Ausscheidungsurographie oder MR-Urographie erfolgen. Beim muskelinvasiven TCC ist die Nachsorge komplexer und abhängig von der Ausgangssituation nach radikaler Zystektomie und Harnableitung [29].

\section{Nierenzellkarzinom}

Nach operativer Therapie des RCC empfehlen die europäischen Leitlinien eine Risikostratifizierung mittels integrierter Stagingsysteme, die sowohl das TNMSystem als auch den Performance-Score oder andere Parameter einbeziehen. Exemplarisch wurde ein solches Nomogramm bereits vorgestellt [16]. Für Erkrankungen mit niedrigem Rezidivrisiko - vergleichbar dem oben vorgestellten kleinen asymptomatischen Tumor empfehlen die Leitlinien ein großzügigeres Nachsorgeschema. Bei Patienten mit mittlerem und hohem Risiko (vergleichbar dem oben vorgestellten Tumor mit Thrombus in der V. cava inferior) sollte eine intensivierte Nachsorge erfolgen [19].
Bemerkenswert in diesem Zusammenhang ist, dass RCC teilweise sehr lange rezidivfreie Intervalle aufweisen und auch nach deutlich mehr als 5 Jahren noch Rezidive auftreten können [9].

\section{Keimzelltumoren}

In der Nachsorge von KZT muss zwischen lokalisierten Tumoren ohne Metastasierung und metastasierten Tumoren sowie zwischen SKZT und NSKZT unterschieden werden. 
Der NSKZT ohne Metastasen unter aktiver Überwachung wird in den ersten 2 Jahren alle 3 Monate nachgesorgt. Immerhin bestehen bei Patienten mit im CT unauffälligen KZT bei 15-30 \% doch Metastasen.

Die Nachsorge des SKZT ist im Detail anders geregelt.

Bei metastasierten Hodentumoren erfolgen die Nachsorgeuntersuchungen engmaschiger [3].

\section{Worauf sollten Rheumatologen achten?}

Aus Sicht des Urologen sollte der Rheumatologe beim Erstkontakt mit einem Patienten mit Symptomen einer rheumatischen Erkrankung die prinzipielle Möglichkeit eines zugrunde liegenden urologischen Malignoms im Hinterkopfbehalten. Eine systematische Suche nach einem Malignom wird zwar aufgrund der Seltenheit dieser Konstellation nicht empfohlen [21], gerade bei atypischer Präsentation ohne klare Serologie sind aber Fragen nach einer Symptomatik und nach regelmäßigen Vorsorgeuntersuchungen oft sinnvoll. Immerhin können verschiedene rheumatische Symptome paraneoplastisch durch ein urologisches Tumorleiden hervorgerufen werden $[15,21,26]$.

Bei vorbekanntem urogenitalem Malignom muss ein Rezidiv ausgeschlossen werden. Es gibt keine Evidenz, ab wann nach potenzieller Kuration eines urologischen Malignoms eine konventionelle oder biologische Basistherapie der rheumatischen Erkrankung sicher begonnen werden kann. Je nach Tumorentität ist mehr oder weniger vorsichtig, bei kritischer Indikationsstellung und nicht kontrollierbarer rheumatischer Erkrankung aber auch ein relativ früher Einsatz empirisch gerechtfertigt. Bei PCa, die aufgrund ihrer Biologie einen in der Regel eher langsamen Verlauf und keine wesentliche immunologische Komponente haben, ist eine notwendige Therapie oft ohne wesentliche Risikoerhöhung durchführbar. Insbesondere der Einsatz der antihormonalen Therapie ist unkritisch. Auch Kortisonderivate sind teils sogar zur Behandlung des PCa förderlich, während ein Rückschluss auf andere entzündungshemmende Prinzipien bisher nicht möglich ist. Da auch PCa hochmaligne sein können, sollte vor Beginn einer antirheumatischen Therapie ein interdisziplinärer Austausch erfolgen.

\section{》) Ein interdisziplinärer Aus-} tausch sollte vor einer antirheumatischen Therapie erfolgen

Beim TCC ist eine potenziell negative Beeinflussung durch Antirheumatika bei unkontrolliertem Karzinom wahrscheinlicher, da hier Anti-Tumor-Immunphänomene die Regel darstellen [13]. Hier können beim Einsatz von Immuntherapeutika, sei es zur Therapie des TCC oder zur Therapie der rheumatischen Erkrankung, unerwünschte Wechselwirkungen eintreten, denn ein breiter Einsatz von Immuntherapeutika im Rahmen von immunonkologischen Ansätzen steht bevor. Auch weiterhin wird bei Patienten mit nicht muskelinvasivem TCC zur Rezidiv- und Progressionsprophylaxe oft eine Instillationsimmuntherapie mit Bacillus CalmetteGuérin (BCG) durchgeführt. Hierunter kann es in sehr seltenen Fällen zur einer BCG-assoziierten Arthritis im Sinne einer reaktiven Arthritis, theoretisch aber auch im Rahmen einer mykobakteriellen Infektion immunsupprimierter Patienten kommen. Daher ist ein intensiverer Gedanken- und Erfahrungsaustausch zwischen Urologen und Rheumatologen/Immunologen wichtig. Besonders kritisch sollte bei vorbekanntem TCC der Einsatz von Cyclophosphamid überdacht werden, da dieses alleine für sich schon TCC induzieren kann. Patienten, die Cyclophosphamid erhalten, sollten regelmäßig vom Urologen zum Ausschluss von TCC untersucht werden.

Das RCC gilt ebenfalls als immunaktivierender Tumor, der schon vor mehr als 10 Jahren im metastasierten Stadium mit Zytokinen behandelt wurde [4]. Allerdings ist ein gehäuftes Auftreten von Erkrankungen des rheumatischen Formenkreises bei Patienten mit RCC in der Literatur nicht beschrieben. Auch beim RCCliegen mittlerweile vielversprechende Resultate zur Behandlung mit modernen Immunkontrollpunktblockern (PD1-, PD-L1- und CTLA-4-Antagonisten) vor [8]. Hier sollte bei einer Koinzidenz von RCC und Erkrankungen des rheumatischen Formenkreises die Therapie zwischen Uroonkologen und Rheumatologen abgestimmt werden.

Bei KZT fällt der Altersgipfel der Erkrankungen nicht mit den Altersgipfeln der meisten rheumatischen Erkrankungen zusammen. Spondylarthritiden stellen eine Ausnahme dar, sind aber kaum je mit paraneoplastischen Symptomen zu verwechseln. Rheumatische Erkrankungen werden eher später als früh auftreten. Dass sie als Spätfolge von Chemotherapien auftreten, ist unwahrscheinlich, da unter den typischen Spätfolgen der Chemotherapie als einziges bekanntes Problem ein Raynaud-artiges Syndrom auf Bleomycin beschrieben ist [3]. Prinzipiell ist bei einer Anamnese von KZT bei Diagnose einer rheumatischen Erkrankung eine uroonkologische Vorstellung sinnvoll, um insbesondere bei Einleitung immunsuppressiver Therapien das Risiko eines Tumorrezidivs abzuschätzen und den Patienten zu beraten.

Bei palliativer Behandlungssituation eines urologischen Malignoms und koinzidenteller rheumatischer Erkrankung sollte in interdisziplinärer Absprache zwischen Urologen, Rheumatologen und ggf. Palliativmedizinern das Für und Wider der individuell möglichen Ansätze der antirheumatischen Therapie abgewogen werden. Hier wird im Regelfall das Befinden des Patienten Vorrang vor der theoretisch denkbaren schnelleren Progression des Tumors haben.

\section{Worauf sollten Urologen achten?}

Aus rheumatologischer Sicht erscheinen für die Zusammenarbeit 2 Punkte wichtig: Erstens sind rheumatische Erkrankungen schwere, die Lebensqualität dramatisch und das Überleben signifikant reduzierende Erkrankungen, die fast immer therapiert werden müssen. Die aus urologischer Sicht oft attraktiv erscheinende Option, nichts zu tun, besteht in der Regel nicht. Rheumatologen werden immer wieder auch urogenitale Malignome ausschließen müssen, sei es, weil $\mathrm{Pa}$ raneoplasien infrage kommen, sei es, weil pathologische Befunde (wie eine persis- 
tierende Mikrohämaturie) auffallen. Hier sind wir auf urologisches Verständnis und die Zusammenarbeit angewiesen. Da diese Themen für Rheumatologen Randthemen sind, für die wir nur eine geringe Expertise haben, wird häufig der direkte Kontakt helfen, effizienter und fehlerfreier zusammenzuarbeiten.

\section{》) Mögliche Exazerbation rheumatischer Erkrankungen durch Immuntherapeutika}

In naher Zukunft werden, soweit heute schon absehbar, zudem immunonkologische Substanzen zur Behandlung von zumindest TCC und RCC zugelassen werden. Wie auch andere Autoimmunerkrankungen werden rheumatische Erkrankungen beim Einsatz z. B. von CTLA-4-Antikörpern oder PD-1-Antikörpern unter Umständen exazerbieren. Auch Neumanifestationen sind denkbar [10, 28]. Das wird uns in eine völlig andere Situation als bei der klassischen Chemotherapie bringen, die durch Immunsuppression rheumatische Probleme meist wirksam mit behandelt. Neu aufgetretene Gelenkschmerzen und -schwellungen, aber auch andere Symptome, die verdächtig auf eine entzündlich rheumatische Systemerkrankung, z. B. eine Dermatomyositis oder einen systemischen Lupus erythematodes, sind, sollten zur raschen rheumatologischen Konsultation führen.

\section{Interdisziplinärer Ansatz}

Die Autoren sind der Ansicht, dass ein direkter, am besten institutionalisierter Austausch zwischen Urologen und Rheumatologen wünschenswert ist. Schon aufgrund der relativen Häufigkeit der Krankheitsbilder und des zunehmend immer höheren Alters unserer Patienten werden wir mehr Patienten mit urogenitalen Tumoren und rheumatischer Erkrankung sehen. Zudem kommen Paraneoplasien rheumatischer Art bei urologischen Malignomen durchaus vor. Neue Immuntherapeutika im onkologischen Ansatz werden Autoimmunphänomene auslösen und vorbestehende rheumatische Erkrankungen beeinflus- sen. Gerade in Anbetracht der vermutlich noch über Jahre spärlichen Evidenz wird der fachübergreifenden Entscheidungsfindung mehr Gewicht zukommen. Die Erfahrungen in unserer Arbeitsgruppe mit der interdisziplinären Betrachtung waren durchweg positiv. Wir hoffen, dass sich das auch in der täglichen Routine durchsetzen wird.

\section{Fazit für die Praxis}

- Stattgehabte wie neu auftretende urogenitale Malignome sind bei Patienten mit rheumatischen Erkrankungen durch demografischen Wandel zunehmend.

- Neu aufgetretene muskuloskeletale Symptome und Autoimmunerkrankungen können selten auch als Paraneoplasien Hinweis für das Vorhandensein urologischer Malignome sein.

- Die Datenlage zum Sicherheitsprofil von Basistherapien und Immunsuppressiva ist für klare Empfehlungen nicht ausreichend, daher wird eine individuelle Abwägung erforderlich sein. Meist wird eine (konventionelle und/oder biologische) Basistherapie aber nicht zu einer wesentlichen Risikoerhöhung führen.

- Der zunehmende Einsatz von Medikation mit positiv immunmodulatorischer Wirkung bei urologischen Tumorleiden könnte zu Neumanifestationen und zur Exazerbation rheumatischer Erkrankungen führen.

- Eine direkte Kommunikation und Kooperation von Rheumatologen und Urologen/Onkologen in einem interdisziplinären Ansatz z. B. in Form gemeinsamer Fallkonferenzen ist wichtig, um diese Herausforderungen zu bewältigen.

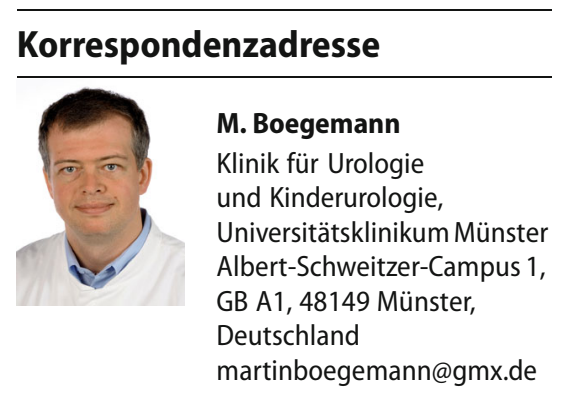

\section{Einhaltung ethischer Richtlinien}

Interessenkonflikt. Dieses Manuskript entstand auf Basis einer von Roche und Chugai unterstützten Arbeitsgruppe (Systemerkrankung RA), aber ohne jegliche Einflussnahme der Firmen auf das Ergebnis oder die Publikation. M. Aringer: Advisory Boards AbbVie, Chugai, MSD, Novartis, Pfizer, Roche. M. Boegemann, R. Alten und S. Zastrow geben an, dass kein Interessenkonflikt besteht.

Dieser Beitrag beinhaltet keine Studien an Menschen oder Tieren.

\section{Literatur}

1. Interdisziplinäre Leitlinie der Qualität S3 zur Früherkennung, Diagnose und Therapie der verschiedenen Stadien des Prostatakarzinoms (2014) AWMF 043/22OL

2. Robert Koch-Institut (Hrsg) und die Gesellschaft der epidemiologischen Krebsregister in Deutschland eV (Hrsg), Berlin (2013) Krebs in Deutschland 2009/2010

3. Albers P, Albrecht W, Algaba F, Bokemeyer C, CohnCedermark G, Fizazi K et al (2015) Guidelines on testicular cancer: 2015 update. Eur Urol 68:1054-1068

4. Allard CB, Gelpi-Hammerschmidt F, Harshman LC, Choueiri TK, Faiena I, Modi P et al (2015) Contemporary trends in high-dose interleukin-2 use for metastaticrenalcell carcinomaintheUnited States. Urol Oncol 33. doi:10.1016/j.urolonc.2015.06.014

5. Andriole GL, Crawford ED, Grubb RL 3rd, Buys SS, Chia D, Church TRet al (2009) Mortality results from a randomized prostate-cancer screening trial. $\mathrm{N}$ Engl J Med 360:1310-1319

6. Babjuk M, Burger M, Zigeuner R, Shariat SF, van Rhijn BW, Comperat E et al (2013) EAU guidelines on non-muscle-invasive urothelial carcinoma of the bladder: update 2013. Eur Urol 64:639-653

7. Blanc PD, Jarvholm B, Toren K (2015) Prospective risk of rheumatologic disease associated with occupational exposure in a cohort of male construction workers. Am J Med 128:1094-1101

8. Bracarda S, Altavilla A, Hamzaj A, Sisani M, Marrocolo F, Del Buono Set al (2015) Immunologic checkpoints blockade in renal cell, prostate, and urothelial malignancies. Semin Oncol 42:495-505

9. Brookman-May S, May M, Shariat SF, Xylinas E, Stief C, Zigeuner R et al (2013) Features associated with recurrence beyond 5 years after nephrectomy and nephron-sparing surgery for renal cell carcinoma: development and internal validation of a risk model (PRELANE score) to predict late recurrence based on a large multicenter database (CORONA/ SATURN Project). Eur Urol 64:472-477

10. Carosella ED, Ploussard G, LeMaoult J, Desgrandchamps F (2015) A Systematic Review of Immunotherapy in Urologic Cancer: Evolving Roles for Targeting of CTLA-4, PD-1/PD-L1, and HLA-G. Eur Urol 68:267-279

11. Eichelberg $C$, Vervenne $W L$, De Santis $M$, von Fischer Weikersthal L, Goebell PJ, Lerchenmuller C et al (2015) SWITCH: a randomised, sequential, open-label study to evaluate the efficacy and safety of sorafenib-sunitinib versus sunitinibsorafenib in the treatment of metastatic renal cell cancer. Eur Urol 68:837-847

12. Ferlay J, Soerjomataram I, Dikshit R, Eser S, Mathers C, Rebelo M et al (2015) Cancer incidence and mortality worldwide: sources, methods and 
major patterns in GLOBOCAN 2012. Int J Cancer 136:E359-86

13. Gakis $G$ (2014) The role of inflammation in bladder cancer. Adv Exp Med Biol 816:183-196

14. Gaston RS, Julian BA, Diethelm AG, Curtis JJ (1991) Effects of enalapril on erythrocytosis after renal transplantation. Ann Intern Med 115:954-955

15. Hakkou J, Rostom S, Bahiri R, Hajjaj-Hassouni N (2012) Paraneoplastic rheumatic syndromes: report of eight cases and review of literature. Rheumatol Int 32:1485-1489

16. Karakiewicz PI, Briganti A, Chun FK, Trinh QD, Perrotte P, Ficarra V et al (2007) Multi-institutional validation of a new renal cancer-specific survival nomogram. J Clin Oncol 25:1316-1322

17. Kim HL, Belldegrun AS, Freitas DG, Bui MH, Han KR, Dorey FJ et al (2003) Paraneoplastic signs and symptoms of renal cell carcinoma: implications for prognosis. JUrol 170:1742-1746

18. Laguna MP, Algaba F, Cadeddu J, Clayman R, Gill I, Gueglio G et al (2014) Current patterns of presentation and treatment of renal masses: a clinical research office of the endourological society prospective study. J Endourol 28:861-870

19. Ljungberg B, Bensalah K, Canfield S, Dabestani S, Hofmann F, Hora M et al (2015) EAU guidelines on renal cell carcinoma: 2014 update. Eur Urol 67:913-924

20. Motzer RJ, Barrios CH, Kim TM, Falcon S, Cosgriff T, Harker WG et al (2014) Phase II randomized trial comparing sequential first-line everolimus and second-line sunitinib versus first-line sunitinib and second-line everolimus in patients with metastatic renal cell carcinoma. JClin Oncol 32:2765-2772

21. Naschitz JE, Rosner I (2008) Musculoskeletal syndromes associated with malignancy (excluding hypertrophic osteoarthropathy). Curr Opin Rheumatol 20:100-105

22. Parkin DM (2011) 2. Tobacco-attributable cancer burden in the UK in 2010. Br J Cancer 105(Suppl 2):6-13

23. Patel NH, Attwood KM, Hanzly M, Creighton TT, Mehedint DC, Schwaab T et al (2015) Comparative analysis of smoking as a risk factor among renal cell carcinoma histological subtypes. J Urol 194:640-646

24. Pound CR, Partin AW, Eisenberger MA, Chan DW, Pearson JD, Walsh PC (1999) Natural history of progression after PSA elevation following radical prostatectomy. JAMA 281:1591-1597

25. Raldow AC, Zhang D, Chen MH, Braccioforte MH, Moran BJ, D'Amico AV (2015) Risk Group and Death From Prostate Cancer: Implications for Active Surveillance in Men With Favorable IntermediateRisk Prostate Cancer. Jama Oncol 1:334-340

26. Sacco E, Pinto F, Sasso F, Racioppi M, Gulino G, Volpe A et al (2009) Paraneoplastic syndromes in patients with urological malignancies. Urol Int 83:1-11

27. Schroder FH, Hugosson J, Roobol MJ, Tammela TL, Ciatto S, Nelen V et al (2009) Screening and prostate-cancer mortality in a randomized European study. NEngl J Med 360:1320-1328

28. Topalian SL, Hodi FS, Brahmer JR, Gettinger SN, Smith DC, McDermott DF et al (2012) Safety, activity, and immune correlates of anti-PD-1 antibody in cancer. N Engl J Med 366:2443-2454

29. Witjes JA, Comperat E, Cowan NC, De Santis M, Gakis G, Lebret T et al (2014) EAU guidelines on muscle-invasive and metastatic bladder cancer: summary of the 2013 guidelines. Eur Uro 65:778-792

\section{Österreichische Open-Access- Vereinbarung mit Springer}

Das Österreichische Akademische Bibliothekskonsortium (KEMÖ) und der österreichische Wissenschaftsfonds (FWF) haben mit dem Springer Verlag ein weitreichendes Lizenzmodell vereinbart. Im Rahmen des Lizenzmodells "Springer Compact" erhalten die Wissenschaftler teilnehmender österreichischer Institutionen Zugang zu mehr als 2.000 wissenschaftlichen Springer-Fachzeitschriften. Außerdem können diese als korrespondierende Autoren in über 1.600 Hybrid-Zeitschriften Open Access (OA) publizieren. Die Vereinbarung greift, sofern die Open-Access-Gebühren nicht bereits von anderer Seite erstattet werden. Das neue Modell bietet WissenschaftlerInnen hervorragende Möglichkeiten, ihre Forschungsergebnisse in einem qualitativ hochwertigen und breiten Zeitschriftenportfolio Open Access zu publizieren. Sie können sich auf das Publizieren konzentrieren und müssen sich nicht um die finanziellen Rahmenbedingungen und administrativen Anforderungen im Hinblick auf OA-Mandate kümmern. Der Zugriff auf alle Springer-Zeitschriften einerseits und die Möglichkeit des mühelosen Open-AccessPublizierens andererseits sollen die Transformation vom subskriptionsbasierten auf ein Open Access basiertes Publikationssystem beschleunigen und die Sichtbarkeit der österreichischen Forschung weltweit erhöhen.

Wie identifiziert sich der korrespondierende Autor bei Springer?

Im Rahmen des elektronischen „MyPublication"-Prozesses wird die Affiliation des korrespondierenden Autors abgefragt und dieser muss zudem einer Open-Access-Publikation zuzustimmen. Im Nachgang bestätigt die beteiligte Institution, die Richtigkeit der Angaben des korrespondierenden Autors hinsichtlich seiner Affiliation und berechtigt somit zur Teilnahme an Springer Compact.

Welche Beitragsformen umfasst SpringerCompact:

- Original Paper - Standardartikel, in dem üblicherweise neue Ergebnisse präsentiert werden (auch Original Research,
Original Article oder Research Paper genannt).

- Review Paper - Standardartikel, der bereits publizierte Ergebnisse interpretiert.

- Brief Communication - kurzer Artikel, der zur schnellen Veröffentlichung eingereicht wurde und die gleiche Struktur hat wie ein Standardartikel.

- Continuing Education - Artikel, der einen wesentlichen Beitrag zur Weiterbildung darstellt (meist medizinisch).

Andere Beitragsformen, beispielsweise Fallberichte (Case Reports), sind in das Abkommen nicht eingeschlossen.

Welche Institutionen sind beteiligt? Eine Auflistung der beteiligten Institutionen kann abgerufen werden unter: http:// www.springer.com/gb/open-access/ springer-open-choice/springer-compact/ agreements-austrian-authors 